\title{
CNES COMO INSTRUMENTO DE GESTÃO E SUA IMPORTÂNCIA NO PLANEJAMENTO DAS AÇÕES EM SAÚdE
}

\section{CNES as a management tool and its importance for planning health actions}

Marcia Regina Pelissari ${ }^{1}$

1. Bacharel em Economia Doméstica, Especialista em Vigilância Sanitária de Alimentos, Especialista em Gestão em Redes de Atenção à Saúde. Secretaria de Estado da Saúde do Paraná - SESA, Paraná (PR), Brasil. ORCID: https://orcid.org/0000-0002-5656-6893.

CONTATO: Marcia Regina Pelissari | Endereço: Rua Cândido Xavier, 755, ap 61 | Curitiba-PR | E-mail: marciarp65@gmail.com

COMO CITAR: Pelissari MR. CNES como instrumento de gestão e sua importância no planejamento das ações em Saúde. R. Saúde Públ. 2019 Jul;2(1):160-166.

(c) COPYRIGHT Esta obra é disponibilizada nos termos da Licença Creative Commons - 4.0

\begin{abstract}
RESUMO Este artigo objetiva descrever as várias maneiras como o Cadastro Nacional de Estabelecimentos de Saúde (CNES) foi aplicado como instrumento de gestão, utilizando a base de dados BVS, SciELO e LILACS. O CNES surgiu em 2001 para suprir as deficiências no cadastro dos Sistemas de Informação Ambulatorial e Hospitalar. Além de atender o proposto, atualmente o CNES é um documento público e sistema de informação oficial de cadastro de todos os estabelecimentos de saúde no país. Mostra-se confiável e com relevância de dados podendo desenvolver estudos no tocante à capacidade instalada e mão-de-obra assistencial. Contudo, é necessária a conscientização dos gestores em mantê-lo atualizado, pois é através do banco de dados do CNES que os governantes são instrumentalizados com informações capazes de subsidiar decisões baseadas em evidências para o planejamento das ações em saúde.
\end{abstract}

PALAVRAS-CHAVE: Instalações de Saúde. Sistemas de Informação em Saúde. Gestão da Informação. 
ABSTRACT This paper aims to describe the various ways of how the National Registry of Health Facilities (CNES) was applied as a management tool, using BVS, SciELO and LILACS database. The CNES was arose in 2001 to fill the deficits on the register of Hospital and Outpatient Information Systems. Additionally of this purpose, currently the CNES is a public document and the official registry information system of all health facilities in the country. It shows itself to be reliable and with data relevance, being able to develop studies related to the installed capacity and health labor workforce. However, it is necessary the manager's concern about keeping it updated, since it is through the CNES database that governors are supplied with information able to support evidence-based decisions for planning health actions.

KEYWORDS: Health, Facilities. Health Information Systems. Information Management.

\section{INTRODUÇÃO}

$\mathbf{A}$ gestão do Sistema Único de Saúde (SUS) é formada por vários instrumentos visando auxiliar o gestor na tomada de decisão para a implementação das Políticas de Saúde. Os Sistemas de Informação em Saúde (SIS) agregam ferramentas importantes para o planejamento e a avaliação das ações em saúde, assim como dos serviços, redes e sistemas de saúde.

No Brasil, mesmo antes da implantação do SUS, diversos SIS foram instituídos, a maior parte de acesso ao público e administrados pelo Ministério da Saúde (MS) através do Departamento de Informática do Sistema Único de Saúde (DATASUS), e tem orientado a condução de estudos que abordam a análise de parâmetros epidemiológicos, sanitários, de aparelhamento e infra-estrutura de oferta de serviços.

O CNES é um dos mais de 400 (quatrocentos) sistemas implantado pelo MS e surgiu com o objetivo de ser base para se conhecer a oferta de serviços de saúde instalada em todo o território nacional. Do mesmo modo, para contribuir na padronização dos dados relativos à saúde de forma que os sistemas de informações que dependam de dados de estabelecimentos de saúde e mão-deobra assistencial encontrem no CNES informações necessárias à execução de seus processos operacionais.

Com o passar dos anos, o CNES tornouse a principal fonte de informação da rede física assistencial no país e é a base para obter dados sobre a quantidade e tipo de serviços oferecidos, 
localização, características de recursos humanos, habilitações, infraestrutura, dentre outros. Sua estrutura de dados fornece suporte para todos os sistemas de informação que utilizem dados relativos a estabelecimentos de saúde.

Atualmente o CNES não é somente uma interface de consulta de cadastro dos estabelecimentos de saúde. Com o decorrer dos anos incorporou uma enorme gama de funcionalidades relacionadas à gestão que de acordo com o Art. 359 da Portaria de Consolidação n 1 de 28/09/20171, o CNES se constitui como documento público e sistema de informação oficial de cadastro de informações de todos os estabelecimentos de saúde no país, independente da natureza jurídica ou integração com o SUS.

O conjunto de informações obtidas neste trabalho permitiu comprovar que os dados do CNES são importantes para a área de planejamento, controle e avaliação em saúde, porém deveriam refletir a real situação do sistema de saúde. Pode-se observar que alguns estudos realizados abordaram elementos onde demonstraram as inconsistências na base de dados do CNES?

O cadastramento e a manutenção dos dados cadastrais no CNES são de obrigação dos estabelecimentos de saúde através de seus responsáveis técnicos ou administrativos, porém percebe-se que as alterações são pontuais buscando pleitear recursos oriundos de políticas públicas ou são realizadas quando há suspensão dos recursos financeiros por inconsistências no cadastro.

Assim, esse estudo tem o propósito de descrever as diversas possibilidades de aplicação onde o CNES foi utilizado como instrumento de gestão, relatando sua implantação, a confiabilidade dos dados, a distribuição e composição dos profissionais e equipamentos e as divergências encontradas, afirmando a necessidade de implementar estratégias e incentivos para melhorar a confiabilidade de dados.

\section{METODOLOGIA}

Trata-se de uma Revisão Bibliográfica Descritiva de Literatura sobre o Cadastro Nacional de Estabelecimentos de Saúde, sistema de informação oficial de cadastramento de informações de todos os estabelecimentos de saúde, que foi desenvolvido em plataforma DELPHI.

Para a busca de artigos publicados em revistas científicas foram consultadas as bases de dados BVS (Biblioteca Virtual de Saúde Pública), SciELO (Scientific Electronic Library Online), ENSP (Escola Nacional de Saúde Pública Sérgio Arouca) e OPAS (Organização Pan-Americana em Saúde), onde utilizando-se os descritores "cadastro", "estabelecimento de saúde", "sistema de informação em saúde", "gestão de informação" e "sistemas de informação" foram identificados 150 artigos e apenas 6 (seis) descreviam a aplicação do CNES no estudo. Devido ao restrito número de artigos, foram utilizadas as publicações dos últimos 6 (seis) anos e uma dissertação publicada em 2004. assim como o periódico do MS contextualizando o histórico do CNES, as normativas que regem os SIS, além da experiência de 7 (sete) anos da autora sua trajetória, apresentada sua importância através dos artigos selecionados e sinalizada a relevância do CNES como fonte de informação para o planejamento das ações em saúde.

\section{RESULTADOS}

Em 1997, o Tribunal de Contas da União TCU elaborou o Relatório do Programa de Ação na Área da Saúde, de proposta do Ministro Relator Humberto Guimarães Souto, sugerindo que fosse elaborado um "programa de ação, contemplando providências de curto e médio prazo, que permitisse", dentre outros atos, "a realização de um amplo diagnóstico da área da saúde e introduzir mecanismos para o acompanhamento, controle e 
avaliação da aplicação dos recursos da área da saúde"3.

Uma das razões para o Relatório do TCU ser elaborado foram as deficiências nos cadastros do Sistema de Informação Ambulatorial - SIA e Sistema de Informação Hospitalar - SIH, no tocante à sua falta de atualização, baixa qualidade e informações incompletas, além de possiveis fraudes cadastrais para aprovação de faturamento ${ }^{4}$, conforme parte do texto transcrito:

Atualmente, o SUS utiliza como instrumentos de gestão as informações cadastrais das unidades de saúde integrantes dos Sistemas de Informação Ambulatorial e Hospitalar (SIA/SUS e SIH/SUS). Sobre esses sistemas, os exames realizados levaram à constatação de que pelo menos 54\% dos municípios visitados e $33 \%$ dos estados não efetuam o cadastramento dos prestadores de serviço com base em estudo sobre a necessidade do serviço ofertado.

Além disso, constatou-se que 56\% dos municípios e $67 \%$ dos estados não realizam a verificação periódica da capacidade instalada dos prestadores de serviço ${ }^{3}$.

O Ministério da Saúde criou a Ficha de Cadastro de Estabelecimentos de Saúde - FCES com o propósito de identificar as dimensões ambulatoriais e hospitalares necessárias ao faturamento para o SUS. Após a criação do Banco de Dados Nacional de Estabelecimentos de Saúde, o cadastro tomou uma proporção cada vez maior. e passou a ser fonte de informação da rede física dos serviços de saúde em todo o território nacional com a estrutura de dados que dá suporte aos demais sistemas de informação ${ }^{4}$.

O processo de recadastramento nacional informatizado foi implementado efetivamente no território nacional apenas em 2003 devido a muitos ajustes no sistema, com o objetivo de tornar o processo totalmente viável para todos os gestores envolvidos ${ }^{4}$.

Do objetivo de ser a base para se conhecer a oferta de serviços de saúde em todo o território nacional, o CNES passou a ser a base para mais de 80 aplicativos. Além de atender o proposto que era para confrontar as informações cadastrais das unidades de saúde integrantes dos sistemas SIA e SIH, atualmente é a base cadastral para operacionalização de diversos sistemas, tais como: Sistema de Regulação (SISREG), Sistema de Informações de Mortalidade (SIM), Fundo Nacional de Saúde (FNS), Sistema de Gerenciamento de Informações Financeiras (SGIF), Sistema e-SUS Atenção Básica, Sistema de Informação do Câncer (SISCAN), Sistema CADSUS WEB, Sistema de Vigilância Alimentar e Nutricional (SISVAN), Sistema de Informação de Nascidos Vivos (SINASC), Sistema de Informação de Agravos de Notificação (SINAN).

Além de incorporar uma gama de funcionalidades relacionadas à gestão, o CNES realizou adequações de regras através do consumo de outros sistemas oficiais, onde se destacam: as regras do CNPJ com a obtenção de dados da Receita Federal do Brasil (RFB), cruzamento dos cadastros dos profissionais médicos nos registros ativos no Conselho Federal de Medicina (CFM). além da revisão dos CPFs inválidos ou inativos.

Em se tratando de fornecer informações para os gestores, pesquisadores, trabalhadores e sociedade em geral, Tomasi e Rizzotto ${ }^{5}$ fizeram análise da distribuição e composição profissional dos Núcleos de Apoio à Saúde da Família (NASF) no Paraná, com dados coletados do CNES nos meses de maio e junho de 2012, pois é no sistema de cadastro onde são cadastrados os tipos de equipe e profissionais com a carga horária determinada pela normativa que rege os NASFs. O estudo demonstrou que apenas 31 dos 399 municípios do Estado do Paraná possuíam NASF implantado e foi considerado pelos autores que havia desigualdade na distribuição e composição profissional, com privilégio para os centros urbanos, ou seja, maior concentração de equipes NASF nos grandes centros urbanos ou em regiões próximas a estes. Atualmente, nos dados atualizados do CNES em 
outubro/2018, constam cadastradas 303 equipes NASF em 252 municípios do Paraná.

Vários levantamentos podem ser realizados com o Banco de Dados do CNES (BDCNES) assim como os vínculos de trabalho no setor saúde que foram analisados na macrorregião Oeste do Estado do Paraná. Eberhardt ${ }^{6}$ identificou a presença de 10.330 vínculos precários, o que representou
36,46\% do universo da pesquisa. Segundo o MS, trabalho precário é aquele realizado sem a proteção social do trabalhador. No CNES, o vínculo precário é cadastrado nos estabelecimentos como Autônomo, Bolsa, Cargo Comissionado, Consultoria, Contrato por prazo determinado, Contrato Verbal/ Informal, Cooperativa, Estágio e Residência.

Figura 1 BDCNES - base de suporte para outros sistemas de informação

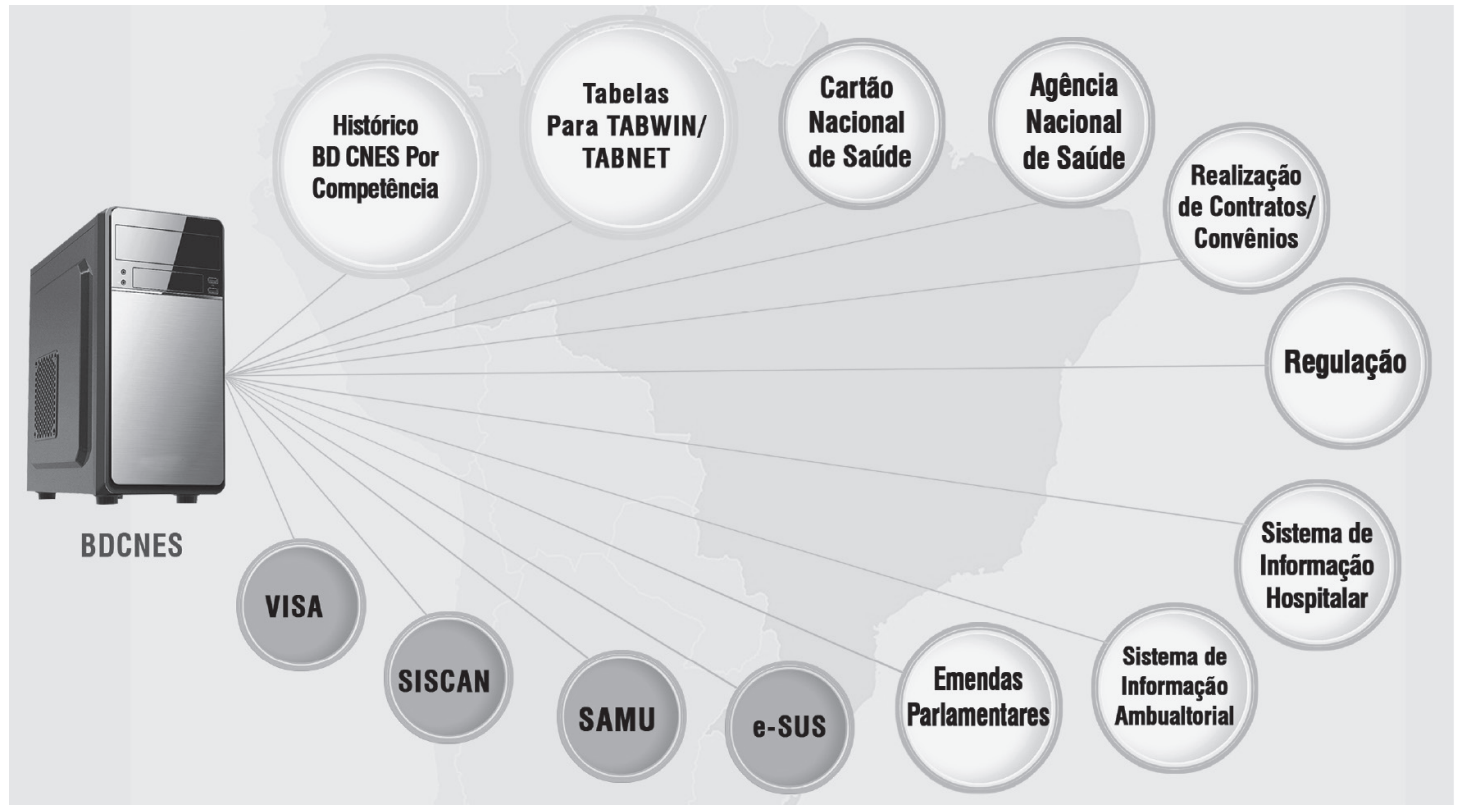

Fonte: Elaborado pela autora (2019)

Quanto aos equipamentos, deve-se salientar que existe uma lacuna entre a aquisição e o registro no CNES. No estudo realizado por Amorim, o MS desenvolveu a ferramenta SomaSUS para auxiliar os gestores na aquisição de Equipamentos MédicoHospitalares (EMH). Embora não seja o foco desses manuais, orientações para o cadastramento no CNES não foram abordadas em nenhum volume analisado.
Mesmo que tivesse, não haveria como cadastrá-los uma vez que atualmente no SomaSuS há uma lista de 532 (quinhentos e trinta e dois) equipamentos e o CNES tem uma relação de apenas 81 (oitenta e um) equipamentos. Isso comprova a pesquisa de Rocha et $\mathrm{Al}^{2}$ onde nas visitas realizadas haviam mais equipamentos avaliados pelo estudo em cada hospital do que aqueles existentes no CNES. 
No que diz respeito à confiabilidade dos dados, Rocha et $\mathrm{Al}^{2}$ utilizaram a BDCNES para comparar um grupo de dados cadastrados. Os 2.777 hospitais visitados ofereceram dados sobre equipamentos, localização geográfica, status de funcionamento e número de leitos. Comparando os hospitais visitados com os registros no CNES, o status de funcionamento estava atualizado em 89\%, o número de leitos em 44\%, no quantitativo de equipamentos $82 \%$ mantinham-se corretos e $63 \%$ estavam com as coordenadas geográficas corretas. Podemos observar que dos dados pesquisados, em 1.222 hospitais o número de leitos não apresentava concordância. Vale lembrar que os leitos hospitalares são componentes essenciais da assistência hospitalar que é organizada a partir das necessidades da população, e as informações precisas instrumentalizam os gestores em saúde com dados capazes de subsidiar decisões baseadas em evidências. Segundo Limå, "a garantia de uma informação de qualidade é condição essencial para a análise objetiva da situação sanitária, para tomada de decisões baseadas em evidências e para a programação de ações de saúde".

No entanto, é hipotético presumir que exista uma base de dados seja ela qual for completamente livre de erros, mesmo realizando rigorosas auditorias. De qualquer forma, a utilização dessas bases em pesquisa pode ser de grande valia para aprimorar a qualidade do cuidado e do sistema de informação 9 .

\section{DISCUSSÃo}

Com base nos artigos selecionados foi possível verificar a importância do CNES para a tomada de decisões e planejamento na área da saúde. Porém é necessário criar estratégias para incentivar o preenchimento correto e o envio de dados com a periodicidade determinada pelo MS. Inconsistências e não envio de base devem ser objetos de monitoramento contínuo ${ }^{9}$.
Rocha et $\mathrm{Al}^{2}$ abordam as inconsistências encontradas no número de contratos registrados e as divergências no cadastro como, por exemplo, estabelecimentos de Alta Complexidade em Oncologia, onde não apresentavam serviços de quimioterapia, radiologia ou cirurgia oncológica. Apenas a exigência de atualização dos dados de acordo com a Portaria SAS/MS n ${ }^{0}$ 118/2014 ${ }^{10}$. onde se define uma periodicidade de 6 meses para atualização, é uma estratégia incapaz de assegurar a real situação. Além disso, é necessária a criação de regras no aplicativo CNES para que o gestor responsável pela homologação dos dados possa perceber a alteração no cadastro e se a mesma está coerente.

Da mesma forma, a Agência Nacional de Saúde (ANS) deve elaborar normativas para que os prestadores não SUS sejam repreendidos quando não atualizam seus cadastros, uma vez que atualmente fazem registro no CNES para cumprir exigência. Assim como os administradores locais do sistema devem criar uma rotina de auditoria, uma vez que o preenchimento correto condizente com a realidade fortalece e qualifica as análises habitualmente realizadas.

\section{CONCLUSÃO}

Os SIS podem ser definidos como um conjunto de componentes inter-relacionados que coletam, processam, armazenam e distribuem as informações para apoiar o processo de tomada de decisão e auxiliar no controle das organizações em saúde 4 .

Diante dos dados levantados, percebe-se que o CNES é uma ferramenta fundamental para uma gestão eficaz e eficiente, proporcionando ao gestor o conhecimento da realidade da rede assistencial e mão-de-obra existente e suas potencialidades auxiliando no planejamento em saúde em todas as esferas administrativas do Governo. 
Tem como finalidades cadastrar e atualizar as informações sobre estabelecimentos de saúde e suas dimensões; disponibilizar informações dos estabelecimentos de saúde para outros sistemas de informação; ofertar para a sociedade informações sobre a disponibilidade de serviços nos territórios, formas de acesso e funcionamento, além de apoiar os gestores na tomada de decisão, planejamento e programação em saúde.

Porém, é necessária a conscientização dos gestores em mantê-lo atualizado, uma vez que é através da sua base de dados que os governantes são instrumentalizados com informações capazes de subsidiar decisões baseadas em evidências².
9. Machado JP, Martins M, Leite, IC. Qualidade de bases de dados hospitalares no Brasil: alguns elementos. Rev. bras. epidemiol. 2016 Set:19(3):567-81.

10. Brasil, Ministério da Saúde. Portaria $n^{\circ} 118$, de 18 de fevereiro de 2014. Desativa automaticamente no Cadastro Nacional de Estabelecimentos de Saúde (SCNES) os Estabelecimentos de Saúde que estejam há mais de 6 (seis) meses sem atualização cadastral [Internet]. 2014 [citado 2019 Mar 16]. Disponivel em: http://bvsms.saude.gov.br/bvs/saudelegis/sas/2014/prt0118_18_02_2014. html.

\section{REFERÊNCIAS}

1. Brasil, Ministério da Saúde, Secretaria de Atenção à Saúde, Departamento de Regulação, Avaliação e Controle. Sistemas de informação da atenção à saúde: contextos históricos, avanços e perspectivas no SUS. Brasilia: Cidade Gráfica; 2015.

2. Rocha TAH, Silva NC, Barbosa ACQ, Amaral PV, Thumé E, Rocha JV. Cadastro Nacional de Estabelecimentos de Saúde: evidências sobre a confiabilidade de dados. Ciênc. saúde coletiva. 2018:23(1):229-40

3. Carvalho CA. Cadastro nacional de estabelecimentos de saúde (CNES): seu desenvolvimento, implantação e uma proposta para sua atualização/manutenção [dissertação]. Rio de Janeiro: ENSP: 2004..

4. Brasil, Ministério da Saúde. Portaria de consolidação $n^{\circ} 1$, de 28 de setembro de 2017. Consolidação das normas sobre os direitos e deveres dos usuários da saúde, a organização e o funcionamento do Sistema Único de Saúde [Internet]. 2017 [citado 2019 Maio 9]. Disponível em: http://bvsms.saude.gov.br/bvs/saudelegis/ gm/2017/prc0001_03_10_2017.html.

5. Tomasi ARP. Rizzoto MLF. Análise da distribuição e composição profissional dos Núcleos de Apoio à Saúde da Família no Paraná. Saúde debate. 2013 Set; 37(98): 427-36.

6. Eberhardt LD, Carvalho M, Murofuse NT. Vínculos de trabalho no setor saúde: o cenário da precarização na macrorregião Oeste do Paraná. Saúde Debate. 2015 Mar:39(104):18-29.

7. Amorim AS, Pinto Junior VL, Shimizu HE. O desafio da gestão de equipamentos médico-hospitalares no Sistema Único de Saúde. Saúde Debate. 2015 Jun;39(105):350-62.

8. Lima CRA. Gestão da qualidade de dados e informações dos sistemas de informação em saúde: subsídios para a construção de uma metodologia adequada ao Brasil [tese]. Rio de Janeiro: FIOCRUZ/ENSP: 2010 